\title{
The classification and treatment strategies of post- esophagectomy airway-gastric fistula
}

\author{
Changchun Wang ${ }^{1,2 \#}$, Chengqiang $\mathrm{Li}^{3 \#}$, Xun Yang ${ }^{1,2}$, Weimin Mao ${ }^{1,2}$, Youhua Jiang ${ }^{1,2}$, Jie Wu ${ }^{1,2}$, Qiang Zhao ${ }^{1,2}$, \\ Anqi Ji ${ }^{3}$, Qixun Chen ${ }^{1,2}$, Hecheng $\mathrm{Li}^{3}$, Jinshi Liu ${ }^{1,2}$ \\ ${ }^{1}$ Department of Thoracic Surgery, Cancer Hospital of the University of Chinese Academy of Sciences (Zhejiang Cancer Hospital), Hangzhou, \\ China; ${ }^{2}$ Institute of Cancer and Basic Medicine (IBMC), Chinese Academy of Sciences, Hangzhou, China; ${ }^{3}$ Department of Thoracic Surgery, Ruijin \\ Hospital, Shanghai Jiao Tong University School of Medicine, Shanghai, China \\ Contributions: (I) Conception and design: Q Chen, H Li, J Liu; (II) Administrative support: W Mao, X Yang; (III) Provision of study materials or \\ patients: C Wang, C Li, X Yang, Q Zhao, A Ji; (IV) Collection and assembly of data: C Wang, C Li, Y Jiang, J Wu, Q Zhao, A Ji; (V) Data analysis \\ and interpretation: C Wang, C Li, Q Chen, H Li, J Liu; (VI) Manuscript writing: All authors; (VII) Final approval of manuscript: All authors. \\ "These authors contributed equally to this work. \\ Correspondence to: Jinshi Liu, MD. Department of Thoracic Surgery, Cancer Hospital of the University of Chinese Academy of Sciences (Zhejiang \\ Cancer Hospital), Hangzhou, China; Institute of Cancer and Basic Medicine (IBMC), Chinese Academy of Sciences, Hangzhou, China. Email: \\ liujinshi208@163.com; Hecheng Li, MD. Department of Thoracic Surgery, Ruijin Hospital, Shanghai Jiao Tong University School of Medicine, \\ Shanghai, China. Email: lihecheng2000@hotmail.com.
}

Background: Airway-gastric fistulas (AGFs) are rare but life-threatening complications after esophagectomy for esophageal cancer. Their effective and reasonable management is challenging and still controversial. This study reports the classification and management strategies of post-esophagectomy AGF based on a retrospective analysis of 26 cases in two large volume centers in China.

Methods: Between January 2000 and December 2017, 6,316 consecutive patients with esophageal carcinoma underwent esophagectomy. AGF was verified in 26 patients. The patients with AGF were divided into two types based on the anatomic characteristics of the fistula. Type I was characterized by the presence of fistula orifices in digestive tract that were higher than those in the airway and were treated with conservative management. Type II had both fistula orifices located on the same horizontal plane and were treated with surgical management. Pearson Chi-Square (R software) was used to compare mortality rates.

Results: From January 2000 and December 2017, 26 cases occurred AGF in 6,316 consecutive patients with esophageal carcinoma underwent esophagectomy and the incidence of AGF was $0.4 \%$. Ten of 12 patients with type I AGF survived. Nine of 14 patients with type II AGF died. There was a significantly difference in the mortality rates between patients with AGF type I and II, which was $16.7 \%(2 / 12)$ and $64.3 \%(9 / 14)\left(\chi^{2}=6.003, \mathrm{P}=0.014\right)$, respectively.

Conclusions: AGF may be classified into two types according to the anatomic characteristics. Type I patients may be cured by conservative management and type II patients, require surgical intervention with pedicled tissues flap wrapping of the airway.

Keywords: Airway-gastric fistula (AGF); esophagectomy; esophageal cancer; classification; management strategy

Submitted Feb 20, 2020. Accepted for publication Jun 07, 2020.

doi: $10.21037 /$ jtd-20-284

View this article at: http://dx.doi.org/10.21037/jtd-20-284

(C) Journal of Thoracic Disease. All rights reserved. 


\section{Introduction}

The development of airway-gastric fistula (AGF) following esophagectomy is a potentially fatal complication associated with substantial morbidity and mortality (1). It includes both digestive tract and tracheobronchial fistulas. Both fistula orifices must be connected. A simple tracheobronchial fistula after esophagectomy is highly unusual and is normally caused by intraoperative injury or prolonged tracheal intubation (1-3). The development of an anastomotic leak or local gastric conduit ischemic necrosis is normally considered to be the predominant reason for acquired tracheobronchial fistulas following esophagectomy. Therefore, AGF may occur as a consequence of leakage from a gastric conduit or anastomosis with subsequent mediastinitis and inflammatory involvement of the tracheobronchial tree (4-6). The most frequent sign of AGF is coughing after swallowing. The nasogastric tube (NGT) is also unable to create negative pressure in some cases. With the persistent aspiration of digestive fluid, the respiratory system becomes contaminated, which may lead to dyspnea and eventually respiratory failure. If the mediastinum and pleural cavity are infected, a rapidly progressive septic disease is unavoidable.

Despite the severity of AGF, there is no generally acceptable therapeutic approach to the management. The literature are comprised mainly case reports, and, as a consequence, there is no ideal therapeutic principle that can be recommend. General conservative treatments include jejunostomy feeding, fasting, the administration of proton pump inhibitors and NGT drainage (7), bronchoscopy (8-11) or gastroscopy (12) and stent insertion. These treatments have shown to achieve reliable good results in several studies. However, Boyd (13) has argued that airway stenting for AGF should not be considered as a definitive therapy because of a high recurrence rate (39\%). Furthermore, stenting may only provide temporization until surgery could be tolerated. Endoscopic fibrin glue injection (14), as well as a novel endoscopic over-thescope-clip device (15), have also been reported to create an effective seal of the fistula. Successful surgical interventions with primary closure of the fistula (16), redoing of esophagogastric anastomosis (16), pedicled muscle flap transposition $(17,18)$ and esophageal diversion with resection of the gastric conduit (19) have been performed by different authors. In general, surgical re-exploration appears to be more reliable than non-surgical methods. However, most of these studies are hampered by very small numbers, and the situations of the patients with AGF are varies from individual to individual.

Therefore, we investigated the feasibility and the limits of AGF classification and corresponding management strategies in this study. We present the following article in accordance with the STROBE reporting checklist (available at http://dx.doi.org/10.21037/jtd-20-284).

\section{Methods}

\section{Patients}

Zhejiang Cancer Hospital and Ruijin Hospital Ethics Committee approved this retrospective study and waived the need for individual consent. A retrospective study was performed that included a consecutive 6,316 cases who underwent esophagectomy for esophageal cancer between January 2000 and December 2017. Among these, 23 cases were diagnosed with AGF postoperatively. Another three cases with post-esophagectomy AGF were transferred from other hospitals. The patients with AGF were divided into two types based on the anatomic characteristics of the fistula. Type I was characterized by the presence of fistula orifices in digestive tract that were higher than those in the airway and were treated with conservative management. Type II had both fistula orifices located on the same horizontal plane and were treated with surgical management. The patients with AGF were between 51 and 73 years of age, with an average age of $61.2 \pm 5.34$ years, and included 3 females and 23 males. The approaches of esophagectomy, the diagnostic time of AGF and the location of the fistula orifices in the digestive tract and airway, are shown in Table 1. Twelve patients with AGF whose fistula orifices were located in the digestive track and airway in a different horizontal plane, were classified as type I; 14 patients with AGF whose fistula orifices were located in the same horizontal plane were classified as type II. Pearson Chi-Square (R software) was used to compare mortality rates.

\section{Diagnosis of $A G F$}

In addition to the frequent coughing after swallowing, another earliest objective sign of AGF was the failure of the NGT to create negative pressure in some cases. Once the negative pressure ball distal to the gastric tube automatically began to inflate frequently, AGF was clinically suspected, 6 cases failure of NGT to create negative pressure in this study. Endoscopy (including bronchoscopy or/and gastroscopy) and computed tomography (CT) of the chest were performed immediately as long as the patients 


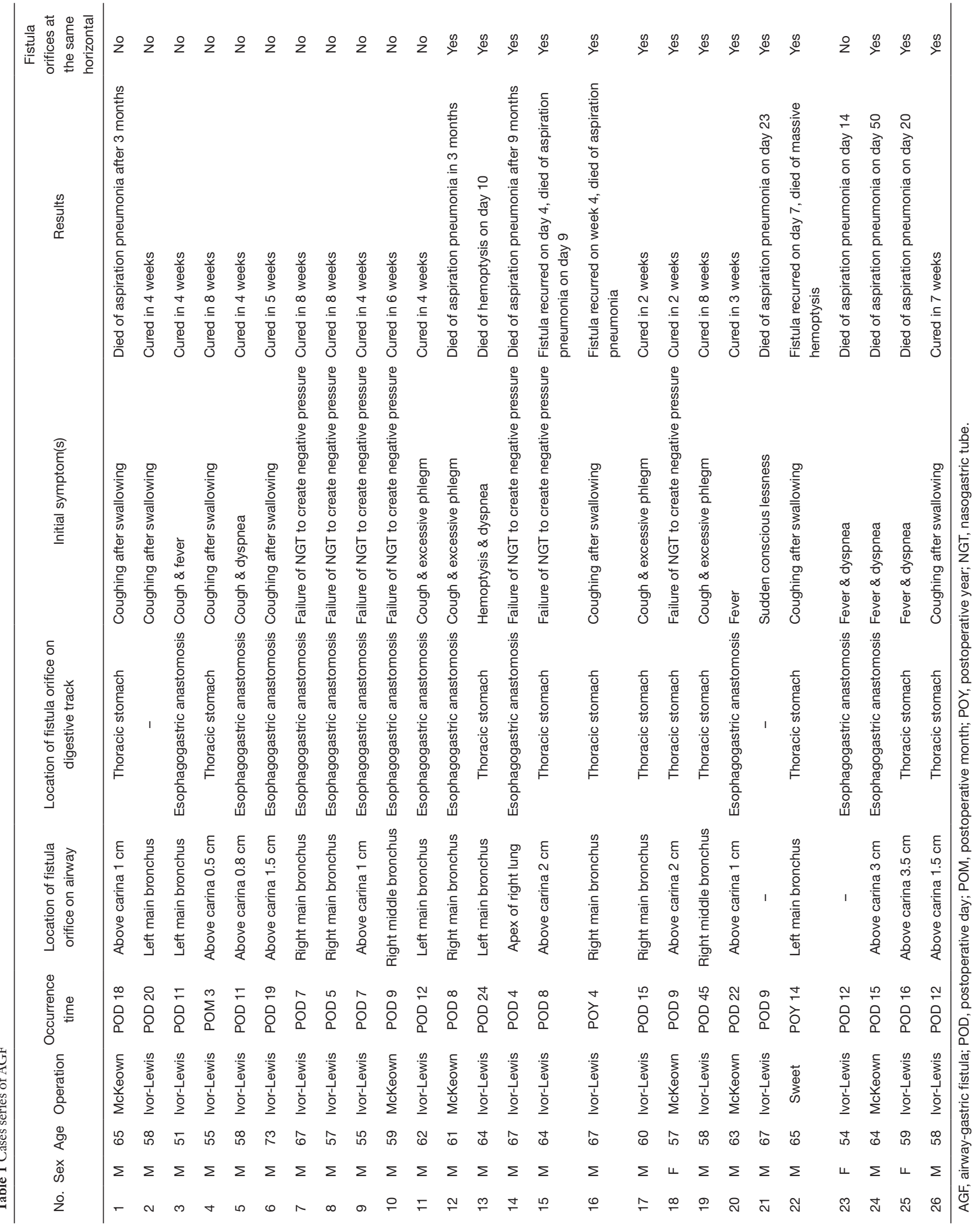



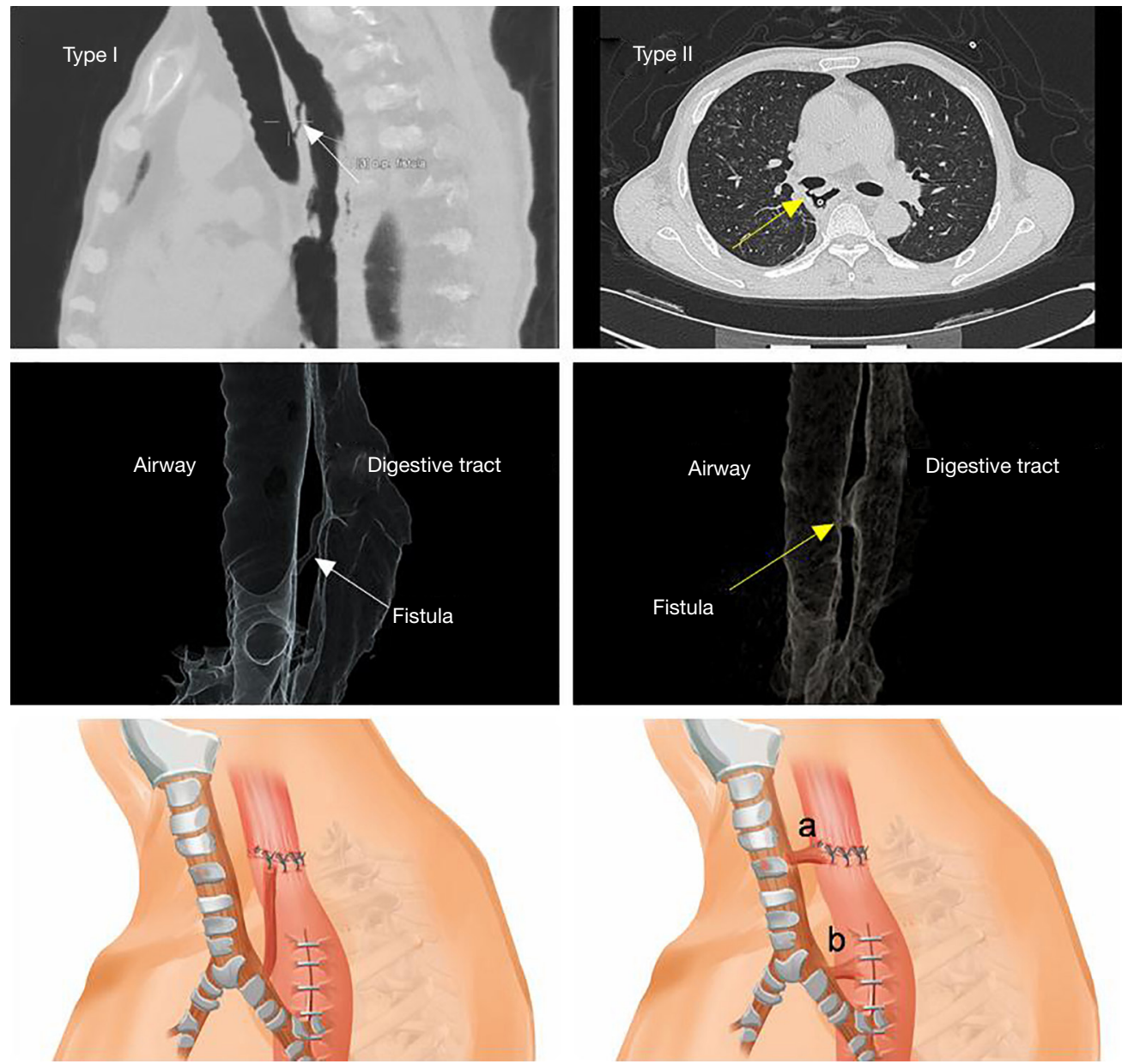

Figure 1 Left side is type I. Fistula orifice in digestive tract is higher than in airway (white arrow). Left diagram shows the type I AGF. Right side is type II. Fistula orifices in digestive tract and that in airway are in the same horizontal plane (yellow arrow). Type IIa, fistula on neck; type IIb, fistula in thoracic(as shown in the right diagram). AGF, airway-gastric fistula.

were able to tolerate the procedures. Gastroscopy was prior to radiographic contrast-medium swallow for the diagnosis of anastomotic leaks because of the ability to directly visualize and examine the anastomosis, quantify the leak and determine of whether or not the gastric conduit was ischemic. Bronchoscopy was carried out to identify the location and size of the airway fistula. A CT scan of the chest was mandatory to rule out the presence of advanced pleural empyema or a mediastinal abscess. A 3D reconstruction of the $\mathrm{CT}$ of the gastric conduit and airway was helpful for determining the appropriate treatment strategy because the size and location of the entire fistula could be directly demonstrated and a determination made about whether or not both fistula orifices were located in the same horizontal plane (Figure 1).

\section{Transnasal transgastric fistula drainage}

If the gastric fistula orifice was located higher than that of the airway fistula, surgery was avoided and double drainage tubes were inserted. One tube was a general NGT, while the other tube was a transnasal transgastric fistula drainage tube with a diameter of $2 \mathrm{~mm}$, which was inserted into the fistula by gastroscopy. Both tubes were connected with a negative pressure ball (Figure 2A). Approximately 4 weeks later, the fistula drainage tube was gradually withdrawn $1 \mathrm{~cm}$ every 3 days. Healing of the fistula was implied if both tubes were able to create negative pressure. 

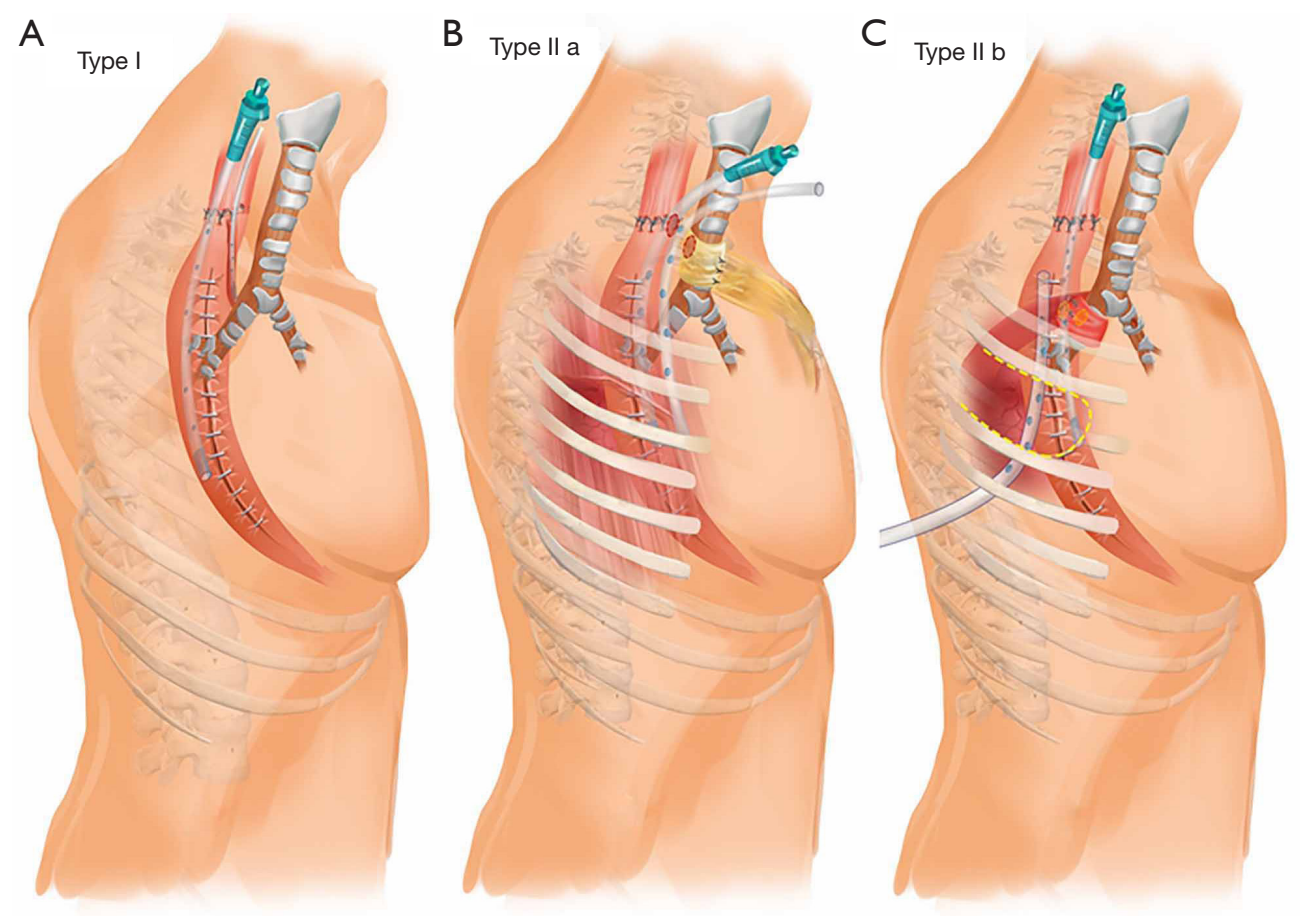

Figure 2 Diagrams show the various managements of different types of AGF. (A) Type I, nasogastric tube + transnasal transgastric fistula drainage; (B) type IIa, pedicled subcutaneous fascia flap wrapping trachea + mediastinum drainage + trans fistula orifice gastrostomy; (C) type IIb, intercostal muscle flap wrapping bronchus/trachea + thoracic tube + nasogastric tube + thoracic gastric fistula orifice suturing (or trans fistula orifice gastrostomy). AGF, airway-gastric fistula.

\section{Surgical airway repairing with pedicled tissue flap}

Surgical repair of the airway was recommended if the both fistula orifices were located in the same horizontal plane. In these cases, two types of pedicle tissue flaps were used according to the location of the fistulas. If the fistula orifices were located on the neck (type IIa), a pedicled subcutaneous fascia flap was appropriate.

With the patient in the supine position, a U-shape incision was made in the anterior cervical neck and median sternotomy to the 2 nd rib. The end of the flap mandatorily needed to exceed the mid-sternum by at least $3 \mathrm{~cm}$. An appropriate size pedicled subcutaneous fascia flap was then created. The epidermis and dermis of the distal $5 \mathrm{~cm}$ of the flap were resected to wrap the trachea without skin. After adhesiolysis, cervical esophagus and the upper gastric conduit were freed until both fistula orifices were identified. The trachea with the fistula orifice was tightly wrapped with the flap. The edge of the flap was sutured to the trachea to avoid slippage. A drainage tube was inserted into the gastric conduit through the fistula orifice without suturing. Another drainage tube was placed into the mediastinum (Figure 2B). The patient was extubated immediately after the operation. The drainage tubes in the gastric conduit and mediastinum were gradually removed when digestive leak was resolved.

If the airway fistula orifice was located at the level of the right/left bronchus or lower trachea (type $\mathrm{Ib}$ ), and the both fistula orifices were in the same horizontal plane, an intercostal muscle flap was a reasonable source of tissue for repairing the airway fistula. The pedicled 4th intercostal muscle was commonly used.

With the patient in the left lateral decubitus position, a standard posterolateral thoracotomy incision was made at the superior border of the 5 th rib. The intercostal muscles were incised on the upper edge of the 5th rib, anterior to the costal cartilage and posterior to the costal angle. The 4th rib periosteum was medially dissected and the 4th rib was completely freed and removed. The intercostal muscle was incised at the lower edge of the $3 \mathrm{rd}$ rib and its front end 


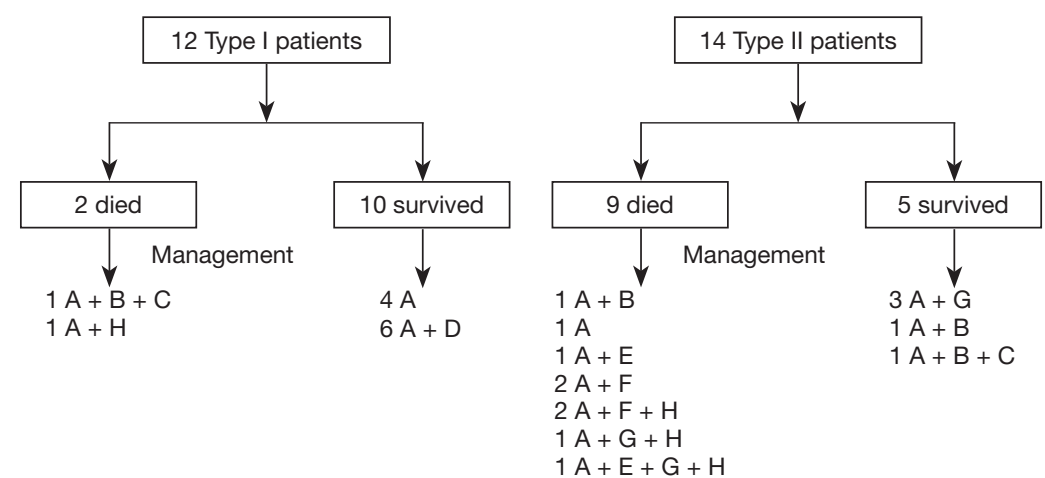

Figure 3 The management AGF patients received. A, fasting + nasogastric tube + jejunostomy and jejunum nutrition + proton pump inhibitor + antibiotic therapy; B, airway stent; C, glue; D, transnasal transgastric fistula drainage; E, thoracic drainage; F, airway and digestive track suturing and pedicled muscle filling; G, surgical airway repairing with pedicled tissue flap wrapping; H, mechanical ventilation. AGF, airway-gastric fistula.

and the intercostal pedicled muscle pleura flap pedicled with the 4th intercostal vessels was isolated. The gastric conduit and the airway were carefully freed around the fistula until both fistula orifices were exposed. The intercostal muscle pleura flap was used to wrap the airway fistula with the pleura towards the airway wall. The edge of the intercostal muscle was sutured to the airway to avoid sliding, and a drainage tube around the fistula was also placed (Figure 2C). The patient was extubated immediately after the operation. Withdrawal of the thoracic drainage tube occurred when the gastric conduit fistula was healed.

\section{Results}

From January 2000 and December 2017, 26 cases occurred AGF in this study and the incidence of AGF was $0.4 \%$. Ten of 12 patients with type I AGF survived. Six of the survivors were treated with transnasal transgastric fistula drainage, four with general conservative management (Figure 3).

Nine of 14 patients with type II AGF died (Figure 3). Four patients who were treated with mechanical ventilation due to severe aspiration pneumonia and respiratory failure died, two were treated with general conservative management, two underwent primary closure of the fistula and insertion of a pedicled muscle flap, and the other patient was treat with an expanding stent. Of those five patients that survived, three were treated with a pedicled tissue flap wrapping the airway fistula, one was treated with an expanding stent and one was cured by temporary airway stenting modified with three-dimensional printing as previous reported (20). There was a significantly difference in the mortality rates for patients with AGF type I and II, which was $16.7 \%(2 / 12)$ and $64.3 \%(9 / 14)\left(\chi^{2}=6.003\right.$, $\mathrm{P}=0.014)$, respectively.

\section{Discussion}

To the best of our knowledge, this is the first classification of AGF into two types according to the anatomic characteristics of the fistula. Type I patients have fistula orifices in digestive tract that are higher than those in airway and type II patients have fistula orifices in the same horizontal plane. Yasuda and his colleagues have classified AGF to three types as anastomotic leakage type, gastric necrosis type and gastric ulcer type (21) that was different from our classification. Following the diagnosis of AGF, the following conservative approaches were applied for all patients: insertion of a NGT to diminish gastric fluid, jejunostomy feeding or nasointestinal feeding to facilitate adequate nutrition, and administration of a proton pump inhibitor to reduce gastric acid output as well as antibiotic therapy when necessary.

Of the 12 patients with type I AGF, four patients were cured with only general conservative approaches to treatment. Stenting was used in one case due to the presence of a severe cough. The patient improved 2 months after stenting and was discharged, but subsequently died of aspiration pneumonia at home in the $3 \mathrm{rd}$ month. One patient died of severe aspiration pneumonia and respiratory failure. Another six patients with type I AGF were 
treated with double tube drainage (NGT plus transnasal transgastric fistula drainage tube). There was no difference in the healing time required between the former four cases and the latter six cases. However, symptoms associated with coughing of gastric fluid were less in the latter group of patients. The transnasal transgastric fistula drainage tube may potentially prevent gastric fluid reflux into the airway and drain the contaminants in time, leading to a faster resolution of the aspiration pneumonia. This was confirmed on CT images as well as by the patients' symptomatic improvement.

Patients with type II AGF were the first to have the airway repaired to avoid an aspiration pneumonia. Of the five patients who survived with type II AGF, three were treated with a pedicled tissue flap wrapping the airway, and two was treated with a stent. Of the nine patient deaths in this group, four patients received mechanical ventilation, two patients had the fistula orifices primarily sutured, the gap between the airway and digestive track filled with a pedicled tissue flap, two patients were treated conservatively, and one patient was treated with a stent. In our experience, stitching the airway fistula orifices was ineffective, while wrapping the airway with a pedicled tissue flap was relatively successful as previously reported (22-24). It was very difficult to free the gastric conduit from the mediastinum, especially when the repair work was carried out more than 2 weeks from the initial operation. The fistula orifices may have been enlarged when the surgeons attempted to free the gastric conduit. In this situation, since a gastric fistula can be healed with a gastrostomy and mediastinum drainage, gastrostomy through the fistula orifices is recommended for most gastric fistulas in the thoracic and all gastric fistulas in the neck. Some authors reported that fistulas can be healed by stitching both fistula orifices and filling using a pedicle muscle flap $(16,17)$. We attempted this approach in two patients with type II AGF and failed. Another two patients who died with type II AGF were only treated conservatively because they refused reoperation (including one patient with a right lung apical defect following gastroesophageal anastomosis leakage).

The severity of the aspiration pneumonia depends on the extent of airway contamination by digestive fluid. The factors that affect the degree of airway pollution may be: (I) the efficiency of gastric emptying and the quantity of gastric fluid; (II) the size of the fistula; (III) whether or not the fistula orifice in the digestive tract and that in the airway are in the same horizontal plane. Gastric emptying may play a primary role. Normally, the stomach is divided into conduits with a diameter of $3-4 \mathrm{~cm}$. The amount of digestive fluid is much less than that of the entire stomach. If the functional capacity of gastric emptying is good and the cough reflex is normal, there will be very little digestive fluid reflux into the airway, and therefore, serious aspiration pneumonia is unlikely to develop. In this study, patient No. 14 died 9 months after conservative treatment, and patient No. 19 underwent a second operation 13 months after the first surgery. This suggests that some patients with type II AGF have enough time to rescue. If a disorder of gastric emptying occurs, aspiration pneumonia may be very serious, because a large amount of gastric fluid will reflux into the airway when the patient coughs. In this situation, an emergency stent insertion may be a potentially helpful approach.

This study has some limitations. We did not analyse the risk factors for AGF. It was not feasible to verify the exact causes associated with AGF such as intraoperative tracheal injury, postoperative mediastinitis, radiation necrosis, erosion injury, intraoperative energy instruments and risk of thrombosis in the consecutive 6,316 cases. As a retrospective study that covered a period of 18 years, the treatment approaches evolved progressively and often mirrored methods reported in the literature. Consequently, different approaches were used to treat patients with the same AGF type. For the use of airway stents, we only had an experience with four patients. Two patients with type II AGF were healed. Some authors claim that the patients with AGF can benefit from early stent placement $(10-12,25)$. However, based on the results from a Mantel-Haenszel analysis, Boyd concluded that though airway stenting for AGF was associated with an initial closure rate of $75 \%$, there was a high recurrence rate of $39 \%$. Therefore, stenting may only provide temporization until surgery can be tolerated and should not be considered as a definitive therapy $(13,26)$.

In conclusion, we first propose a classification and treatment strategies for AGF based on the anatomical characteristics of the fistulas. For patients with type I AGF, the digestive tract fistula orifice is higher than that of the airway fistula; most patients can be cured in approximately 8 weeks using the method of double tube drainage. For patients with type II AGF, the digestive tract fistula orifice and the airway fistula orifice are in the same horizontal plane; a pedicled tissue flap wrapping the defect airway is recommended. Patients with either type I or II AGF who require mechanical ventilation are likely to have a fatal outcome. Second, we put forth the principle of protecting 
the airway first. With adequate enteral and parenteral nutrition support, the mortality rate from digestive tract fistula has decreased significantly. If the airway is well repaired and protected, the airway digestive tract fistula can be converted into a simple digestive tract fistula. Thus, the treatment becomes relatively easy.

\section{Acknowledgments}

We would like to acknowledge Ocholi Don Maimba for language editing support. We also thank Zhixing Li who serves in the Shanghai Huishu Medical Technology Development Co., Ltd. for drawing the diagrams for this paper.

Funding: This study was supported by the National Key Research and Development Program of China (Project 2017YFC0113500), the Medical and Health of Zhejiang Province Scientific Research Project (2014KYA101, 2013KYA031) and the Zhejiang Provincial Natural Science - Mathematical Medicine Association Joint Fund (LSY19H160001).

\section{Footnote}

Reporting Checklist: The authors have completed STROBE reporting checklist. Available at http://dx.doi.org/10.21037/ jtd-20-284

Data Sharing Statement: Available at http://dx.doi. org/10.21037/jtd-20-284

Peer Review File: Available at http://dx.doi.org/10.21037/jtd20-284

Conflicts of Interest: All authors have completed the ICMJE uniform disclosure form (available at http://dx.doi. org/10.21037/jtd-20-284). HL serves as an unpaid editorial board member of Fournal of Thoracic Disease from Jul 2018 to Jun 2020. The other authors have no conflicts of interest to declare.

Ethical Statement: The authors are accountable for all aspects of the work in ensuring that questions related to the accuracy or integrity of any part of the work are appropriately investigated and resolved. The study was conformed to the provisions of the Declaration of Helsinki (as revised in 2013). Zhejiang Cancer Hospital (IRB-2018213) and Ruijin Hospital Ethics Committee (2018-104) approved this retrospective study and waived the need for individual consent.

Open Access Statement: This is an Open Access article distributed in accordance with the Creative Commons Attribution-NonCommercial-NoDerivs 4.0 International License (CC BY-NC-ND 4.0), which permits the noncommercial replication and distribution of the article with the strict proviso that no changes or edits are made and the original work is properly cited (including links to both the formal publication through the relevant DOI and the license). See: https://creativecommons.org/licenses/by-nc-nd/4.0/.

\section{References}

1. Kalmár K, Molnár TF, Morgan A, et al. Non-malignant tracheo-gastric fistula following esophagectomy for cancer. Eur J Cardiothorac Surg 2000;18:363-5.

2. Reed MF, Mathisen DJ. Tracheoesophageal fistula. Chest Surg Clin N Am 2003;13:271-89.

3. Paraschiv M. Tracheoesophageal fistula--a complication of prolonged tracheal intubation. J Med Life 2014;7:516-21.

4. Bartels HE, Stein HJ, Siewert JR. Tracheobronchial lesions following oesophagectomy: prevalence, predisposing factors and outcome. Br J Surg 1998;85:403-6.

5. Bartels HE, Stein HJ, Siewert JR. Respiratory management and outcome of non-malignant tracheo-bronchial fistula following esophagectomy. Dis Esophagus 1998;11:125-9.

6. Maruyama K, Motoyama S, Sato Y, et al. Tracheobronchial lesions following esophagectomy: erosions, ulcers, and fistulae, and the predictive value of lymph node-related factors. World J Surg 2009;33:778-84.

7. Martin-Smith JD, Larkin JO, O'Connell F, et al. Management of gastro-bronchial fistula complicating a subtotal esophagectomy: a case report. BMC Surg 2009;9:20.

8. Schweigert M, Dubecz A, Beron M, et al. Management of anastomotic leakage-induced tracheobronchial fistula following oesophagectomy: the role of endoscopic stent insertion. Eur J Cardiothorac Surg 2012;41:e74-80.

9. Wang CY, Chou CH, Wang HP, et al. Successful treatment of bronchoesophageal fistula with esophageal and bronchial stenting. J Formos Med Assoc 2011;110:270-2.

10. Shin JH, Kim JH, Song HY. Interventional management of esophagorespiratory fistula. Korean J Radiol 2010;11:133-40.

11. Li ZM, Lu HB, Ren KW, et al. Thoracic stomach-right main bronchus fistula treated with dual Y-shaped covered 
airway stents. Clin Radiol 2017;72:517.e1-6.

12. Hugin S, Johnson E, Johannessen HO, et al.

Gastrobronchial fistula following minimally invasive esophagectomy for esophageal cancer in a patient with myotonic dystrophy: case report. Int J Surg Case Rep 2015;17:31-5.

13. Boyd M, Rubio E. The utility of stenting in the treatment of airway gastric fistula after esophagectomy for esophageal cancer. J Bronchology Interv Pulmonol 2012;19:232-6.

14. Tong DK, Law S. Post-oesophagectomy anastomoticbronchial fistula. Hong Kong Med J 2011;17:325-7.

15. Zolotarevsky E, Kwon Y, Bains M, et al.

Esophagobronchial fistula closure using a novel endoscopic over-the-scope-clip. Ann Thorac Surg 2012;94:e69-70.

16. Shi H, Wang WP, Gao Q, et al. Single-stage surgical repair of airway gastric fistula after esophagectomy. J Cardiothorac Surg 2014;9:30.

17. Maruyama K, Motoyama S, Okuyama M, et al. Esophagotracheal fistula caused by gastroesophageal reflux 9 years after esophagectomy. World J Gastroenterol 2007;13:801-3.

18. Morita M, Saeki H, Okamoto T, et al. Tracheobronchial fistula during the perioperative period of esophagectomy for esophageal cancer. World J Surg 2015;39:1119-26.

19. Buskens CJ, Hulscher JB, Fockens P, et al. Benign tracheo- neo-esophageal fistulas after subtotal esophagectomy. Ann Thorac Surg 2001;72:221-4.

20. Han Y, Yang S, Huang W, et al. A hem-o-lok-induced tracheoesophageal fistula cured by temporary airway stenting modified with three-dimensional printing. Ann Thorac Surg 2018;106:e219-21.

21. Yasuda T, Sugimura K, Yamasaki M, et al. Ten cases of gastro-tracheobronchial fistula: a serious complication after esophagectomy and reconstruction using posterior mediastinal gastric tube. Dis Esophagus 2012;25:687-93.

22. Balakrishnan A, Tapias L, Wright CD, et al. Surgical management of post-esophagectomy tracheo-bronchialesophageal fistula. Ann Thorac Surg 2018;106:1640-6.

23. Hayashi K, Ando N, Ozawa S, et al. Gastric tube-totracheal fistula closed with a latissimus dorsi myocutaneous flap. Ann Thorac Surg 1999;68:561-2.

24. Fricke A, Bannasch H, Klein HF, et al. Pedicled and free flaps for intrathoracic fistula management. Eur J Cardiothorac Surg 2017;52:1211-7.

25. Lambertz R, Hölscher AH, Bludau M, et al. Management of tracheo- or bronchoesophageal fistula after Ivor-Lewis esophagectomy. World J Surg 2016;40:1680-7.

26. Sahebazamani M, Rubio E, Boyd M. Airway gastric fistula after esophagectomy for esophageal cancer. Ann Thorac Surg 2012;93:988-90.
Cite this article as: Wang C, Li C, Yang X, Mao W, Jiang Y, Wu J, Zhao Q, Ji A, Chen Q, Li H, Liu J. The classification and treatment strategies of post-esophagectomy airwaygastric fistula. J Thorac Dis 2020;12(7):3602-3610. doi: $10.21037 /$ jtd-20-284 